Notfall Rettungsmed 2022 $25: 75-76$

https://doi.org/10.1007/s10049-022-00994-1

Angenommen: 3. Februar 2022

(c) The Author(s), under exclusive licence to Springer Medizin Verlag $\mathrm{GmbH}$, ein Teil von Springer Nature 2022

\section{Redaktion}

B. Ondruschka, Hamburg

F.T. Fischer, München

\title{
Forensischer Blick auf Morphologie und Dokumentation von Verletzungen sowie Einsätze im Polizeigewahrsam
}

\author{
Gemeinsam voran!
}

\author{
Benjamin Ondruschka ${ }^{1}$ Florian T. Fischer ${ }^{2}$ \\ 'Institut für Rechtsmedizin, Universitätsklinikum Hamburg-Eppendorf, Hamburg, Deutschland \\ ${ }^{2}$ Institut für Rechtsmedizin, Ludwig-Maximilians-Universität München, München, Deutschland
}

\section{Autoren}
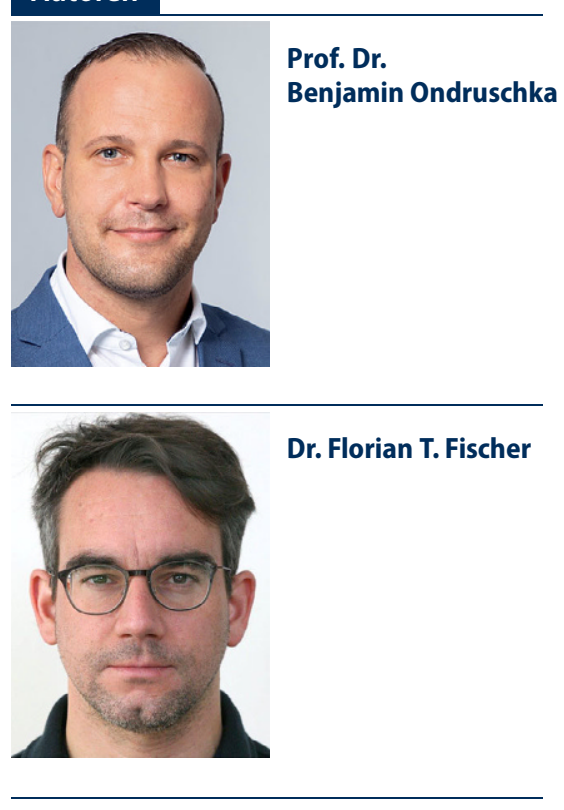

In Ausgabe 8/21 der Notfall+Rettungsmedizin lesen Sie den ersten Teil des spannenden Leitthemas der Heftherausgeber mit dem Schwerpunkt auf Rettungsmaßnahmen, Todesfeststellung und Medizinrecht.

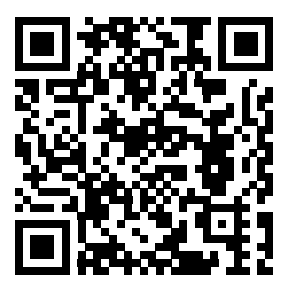

QR-Code scannen \& Beitrag online lesen
In unseren Händen lag bereits eine Ausgabe der Notfall+Rettungsmedizin zu Fragestellungen an der Schnittfläche zwischen Notfallmedizin einerseits und Rechtsmedizin andererseits. Zweifelsfrei können dabei mit dem ersten Aufschlag noch nicht alle relevanten und assoziierten Themenfelder abgearbeitet sein. Umso erfreulicher ist die Möglichkeit, mit der nunmehr vorliegenden zweiten Ausgabe weitere Leitthemenbeiträge für die interessierte Leserschaft bereitzustellen - kompakt, aktuell und kompetent aus der Feder von Expert:innen. Ziel der vorliegenden Zusammenstellung soll ein Bestärken in der Handlungssicherheit auf der Straße und in den Notaufnahmen sein, wenn Sie im Kontext von strafprozessualen Ermittlungsverfahren als Ärzt:innen, Pflegekräfte und Rettungsteams an der Schnittstelle der Fächer agieren.

M. Windgassen et al. berichten in „Todesfälle durch scharfe Gewalt in Berlin"von einer epidemiologischen Auswertung aller in der Berliner Rechtsmedizin zur Untersuchung gelangten Delikte durch Einsätze von Stichwaffen. Die Arbeit zeigt zeit- und stadtvierteltypische Anhäufungen, die in Metropolregionen der BRD sicher reproduzierbar wären und uns allen aus dem praktischen Alltag bekannt sein dürften. In entsprechenden Fällen ist neben der Akutversorgung der Verletzungen für die Strafermittlungsbehörden insbesondere rele- vant, ob diese Verletzungen eine abstrakte oder konkrete Lebensgefahr begründen können. Hiervon abhängig kommt es zur Eingruppierung der Straftat für das weitere Ermittlungsverfahren in Abhängigkeit von der Deliktschwere.

L. Knöfel et al. werten medizinische und rechtliche Aspekte des Polizeigewahrsams aus. In dieser besonders heiklen Situation für Betroffene und diesem „fremden Terrain" für die meisten ärztlichen Kolleg:innen geht es zum einen um Aspekte der Beweismittelsicherung, allen voran um unter forensischen Gesichtspunkten und Qualitätsansprüchen durchgeführte Blutentnahmen zur Konzentrationsbestimmung von Alkohol, Drogen und/oder Medikamenten. Zum anderen betrifft es vor allem die notwendigen Kompetenzen in der Bewertung der Gewahrsams- bzw. Verwahrfähigkeit der Proband:innen für einen begrenzten Zeitraum (Beendigung des Polizeigewahrsams - Überführung in die Untersuchungshaftanstalten) im Kontext von psychiatrischen Auffälligkeiten, Intoxikationen oder frischen Verletzungen.

„Spurensicherung und Verletzungsdokumentation in der Präklinik und Notaufnahme" ist ein Übersichtsbeitrag von C. Richter u. R. Lessig zu Fragen der adäquaten Befunddokumentation vor Wundversorgung in Fällen fremder Gewalteinwirkungen. Neben der zumeist annehmbaren konkludenten Einwilligung der von 
Gewalt betroffenen Patient:innen in eine entsprechende gewissenhafte Befunddokumentation ist auch das besondere Tätigkeitsfeld in der Wundversorgung und Spurensicherung an tatverdächtigen Personen relevant. Hier giltes, an die Wahrung der ärztlichen Schweigepflicht zu denken, sofern nicht bereits initial ein Auftrag der Ermittlungsbehörden nach $\S 81$ a Strafprozessordnung vorliegen sollte oder das Durchbrechen der ärztlichen Schweigepflicht zur Wahrung höherer Rechtsgüter im Individualfall erforderlich wird.

\section{I) Auch die Wundversorgung und} Spurensicherung an tatverdächtigen Personen ist relevant

Unsere Kollegen S.N. Kunz u. L.F. Krys erörtern ferner das notfallmedizinisch relevante Thema der Elektroschockdistanzwaffen, welche mehr und mehr bei Einsatzgruppen der Polizei in Deutschland Einzug halten. Neben der puren und sehr charakteristischen Verletzungsmorphologie ist insbesondere die besonders vulnerable Klientel zu berücksichtigen, die in Elektroschockdistanzwaffeneinsätzen getroffen werden dürfte und sich regelhaft in besonderen Ausnahmesituationen und kardiovaskulären Hocherregungszuständen befindet. Die zunehmende Verwendung auch bei uns fordert eine enge Verzahnung zwischen prä- und innerklinischer Versorgung sowie eine gut dokumentierte Einsatzlage mit besonderer Bedeutung in Bezug auf potenzielle Fallstricke in der (späteren) gutachterlichen Praxis bei Komplikationen, Vorwurfslagen oder der Ermittlungsführung in Kapitaldelikten.

Gleichwohl wir mit dem vorliegenden Cuvre keinesfalls Anspruch auf Vollständigkeit in der Verzahnung der beiden Fachbereiche erheben wollen, liefert das Leitthema doch eindrucksvoll den Beleg, dass die Notfallmedizin in relevanten Anteilen nicht ohne rechtsmedizinische Expertise auskommt und dass eine vitale Rechtsmedizin in besonders vielfältiger Weise im Tätigkeitsfeld der Notfallmediziner:innen gefragt war, ist und sein wird.

Prof. Dr. B. Ondruschka

Dr. F.T. Fischer

München und Hamburg, im Januar 2022
Korrespondenzadresse

\section{Prof. Dr. Benjamin Ondruschka}

Institut für Rechtsmedizin, Universitätsklinikum

Hamburg-Eppendorf

Butenfeld 34, 22529 Hamburg, Deutschland

b.ondruschka@uke.de

\section{Dr. Florian T. Fischer}

Institut für Rechtsmedizin, Ludwig-

Maximilians-Universität München

Nußbaumstraße 26, 80336 München,

Deutschland

florian.fischer@med.uni-muenchen.de

Interessenkonflikt. B. Ondruschka und F.T. Fischer geben an, dass kein Interessenkonflikt besteht.

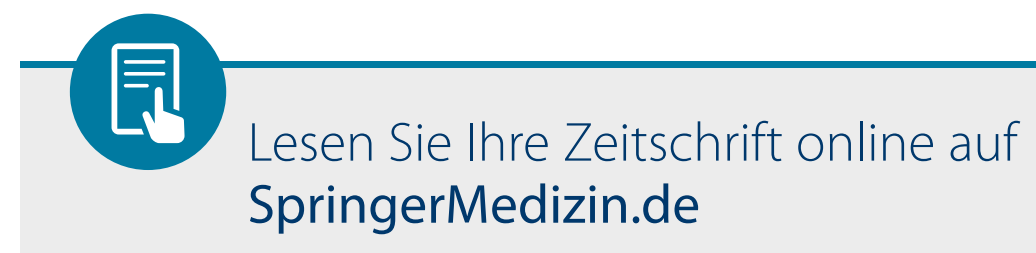

SpringerMedizin.de bietet Ihnen Zugang zu allen elektronisch verfügbaren Ausgaben und dem CME-Angebot Ihrer Zeitschrift - unabhängig davon, seit wann Sie die Zeitschrift abonniert haben.

So einfach erhalten Sie Zugang zum Online-Archiv:

- Registrieren Sie sich einmalig auf www.springermedizin.de/register Geben Sie dabei Ihre Einheitliche Fortbildungsnummer (EFN) an.

- Ihr Benutzername entspricht Ihrer E-Mail-Adresse, Ihr Passwort können Sie frei wählen und später jederzeit unter "Mein Profil“ ändern.

- Falls Sie bereits ein (Print-) Abonnement bei uns haben, geben Sie bei der Registrierung die Lieferadresse Ihrer Zeitschrift an. Damit wird Ihr Abo-Zugang auf springermedizin.de freigeschaltet.

\section{Sind Sie bereits bei SpringerMedizin.de registriert?}

Dann wird Ihr Zeitschriftenabonnement automatisch Ihrem Online-Nutzerkonto hinzugefügt. Sollten die Angaben Ihres Online-Accounts nicht eindeutig mit den Angaben Ihres Zeitschriften-Abonnements übereinstimmen, kann die Zuordnung nicht sicher erfolgen. In diesem Fall und bei allen anderen Fragen zum Online-Zugang kontaktieren Sie bitte unseren Kundenservice unter: Kundenservice@springermedizin.de

Telefonisch erreichen Sie die Hotline montags bis freitags von 9.00 bis 17.00 Uhr kostenfrei unter 0800-77 80777 sowie gebührenpflichtig aus dem Ausland unter +4930884293600. 